\title{
Lluvia de semillas, depredación de semillas y germinación de especies nativas en plantaciones de Pinus radiata en Chile centro-sur: efecto de la distancia a bosque nativo y presencia de sotobosque
}

\author{
Seed rain, seed predation and germination of native species in Pinus radiata plantations \\ in south-central Chile: effects of distance to native forest and presence of understory
}

\author{
Valentina García a , Javier Simonetti a*, Pablo Becerra ${ }^{\text {b,c }}$ \\ *Autor de correspondencia: ${ }^{\text {a }}$ Universidad de Chile, Facultad de Ciencias, Departamento de Ciencias Ecológicas, \\ Las Palmeras 3425, Santiago, jsimonet@uchile.cl \\ b Pontificia Universidad Católica de Chile, Facultad de Agronomía e Ingeniería Forestal, \\ Departamento de Ecosistemas y Medio Ambiente, Santiago, Chile. \\ ${ }^{\mathrm{c}}$ Center of Applied Ecology and Sustainability (CAPES), Santiago, Chile.
}

\begin{abstract}
SUMMARY
Native vegetation development in forestry plantations may depend on seed dispersal, seed predation and seed germination. These processes may depend on distance to native forest fragments and presence of understory. In this study we evaluated the effect of distance to native forests on seed rain and seed predation and the effect of presence of understory on seed rain, post-dispersal predation and germination in Pinus radiata plantations of Chile. We installed seed traps, Petri dishes with Quillaja saponaria seeds and sowed seeds of this species at different distances to native forest fragments, in plantations with and without understory. The total number of seeds and species richness in the seed rain was negatively correlated to distance to native forests, although only in plantations with understory. The total number of seeds did not vary between plantations with and without understory, but the species richness in the seed rain was higher in the one with understory. Seed predation was not related to distance to native forests, neither was the presence of understory and germination higher in plantations with understory. These results suggest that regeneration of native woody species in plantations is negatively affected by distance to native forest and positively affected by the presence of understory. Thus, forestry management should consider dispersal distances and preservation of understory in plantations in order to improve the biodiversity conservation in forest landscapes.
\end{abstract}

Key words: forestry plantations, understory, biodiversity, conservation.

\section{RESUMEN}

El desarrollo de vegetación nativa al interior de plantaciones forestales podría depender de la dispersión, depredación y germinación de semillas, y estos de la distancia a bosques nativos y presencia de sotobosque. En este trabajo se evaluó el efecto de la distancia a fragmentos de bosque nativo sobre la lluvia de semillas y la depredación de semillas, y el efecto de la presencia de sotobosque sobre la lluvia de semillas, depredación post-dispersión y germinación en plantaciones de Pinus radiata de la región del Maule de Chile. Se dispusieron trampas de semillas, placas Petri con semillas de Quillaja saponaria, y se sembraron semillas de esta especie a diferentes distancias de fragmentos de bosque nativo en plantaciones con y sin sotobosque. El número de semillas total y riqueza de especies en la lluvia de semillas se correlacionó negativamente con la distancia al bosque nativo, aunque solo en plantaciones con sotobosque. La abundancia de semillas no varió entre plantaciones con y sin sotobosque, pero la riqueza de especies en la lluvia de semillas fue mayor con sotobosque. La depredación de semillas no se relacionó con la distancia al bosque nativo ni presencia de sotobosque, y la germinación fue mayor con sotobosque. Estos resultados sugieren que la distancia al bosque nativo influye negativamente y la presencia de sotobosque positivamente en la regeneración de especies leñosas en plantaciones. El manejo de plantaciones debería considerar las distancias de dispersión y mantención del sotobosque para mejorar la conservación de la biodiversidad en paisajes forestales.

Palabras clave: plantaciones forestales, sotobosque, biodiversidad, conservación.

\section{INTRODUCCIÓN}

Las plantaciones forestales, especialmente aquellas basadas en especies exóticas, han sido consideradas como “desiertos biológicos", capaces de sostener muy poca o ninguna biodiversidad nativa (Brockerhoff et al. 2008). No obstante, en los últimos años ha surgido evidencia que las plantaciones forestales serían capaces de mante- 
ner mayor biodiversidad que lo supuesto (Brockerhoff et al. 2008). Por ejemplo, plantaciones de coníferas exóticas pueden presentar una variada diversidad de especies de plantas (Brockerhoff et al. 2003, Ramírez et al. 1984), y vertebrados nativos, la cual a su vez estaría directamente relacionada a la presencia de sotobosque bajo el dosel de las plantaciones (Simonetti et al. 2012). Además, cuando las plantaciones presentan sotobosque, aumentaría la conectividad entre fragmentos de bosque nativo inmersos en matrices forestales (Brockerhoff et al. 2008).

Los efectos de las plantaciones forestales sobre la biodiversidad se relacionan con el manejo que se haga de ellas (Brockerhoff et al. 2008). En el marco del Convenio sobre Diversidad Biológica, firmado entre otros países por Chile, se espera que al año 2020 las áreas sometidas a uso forestal sean manejadas sosteniblemente para asegurar la conservación de biodiversidad (CBD 1992). Junto a este requerimiento, las empresas forestales deben tener en consideración el creciente interés público por productos generados mediante técnicas más sustentables e implementar medidas para cumplir con metas de sustentabilidad. Más aún, la comercialización de productos forestales puede depender de procesos de certificación que aseguren la conservación de la biodiversidad en paisajes con plantaciones forestales (FSC 2002). Por esto, el estudio acerca de los factores que influyen en la presencia de biodiversidad nativa dentro de plantaciones puede ser crucial para establecer estrategias de manejo que satisfagan estas demandas.

El establecimiento de sotobosque bajo el dosel de las plantaciones forestales depende en primera instancia de que semillas de plantas nativas se dispersen hacia el interior de estas, luego de que sobrevivan a la depredación post-dispersión y posteriormente que germinen. Estos eventos determinan la probabilidad de reclutamiento de una especie, y pueden verse afectados tanto por factores bióticos como abióticos (Grubb 1977). En plantaciones forestales, la distancia al borde colindante con bosque nativo puede afectar la dispersión de las semillas provenientes del bosque, ya que en general la lluvia de semillas es dependiente de la distancia a la fuente de semillas (Utsugi et al. 2006). Por ejemplo, en plantaciones de Cryptomeria japonica (L.f.) D. Don. en Japón la diversidad y frecuencia de la regeneración de especies se redujo con la distancia al bosque nativo y el tamaño de semillas de las especies fue la principal variable explicando esta variabilidad, lo cual sugiere que la dispersión puede limitar fuertemente el reclutamiento (e.g. Gonzales y Nakashizuka 2010). Por otra parte, se esperaría que los depredadores de semillas provengan principalmente del bosque nativo y, por lo tanto, que la depredación de semillas fuera mayor en áreas más cercanas al borde colindante con bosque nativo. Sin embargo, existe evidencia que la depredación de semillas en plantaciones forestales aumenta con la distancia al bosque nativo (Craig et al. 2011). Consistente con esto, la abundancia de aves granívoras ha sido observada mayor en plantaciones de Pinus radiata D. Don. que en el bosque nativo (Vergara y Simonetti 2004), mientras que la abundancia de micromamíferos depredadores de semillas en plantaciones de $P$. radiata no difiere de aquella observada en pequeños fragmentos de bosque nativo, y es incluso mayor en plantaciones que en áreas extensas de bosque nativo (Saavedra y Simonetti 2005). En estos casos sería esperable una mayor depredación de semillas a mayor distancia del bosque nativo al interior de plantaciones de $P$. radiata.

Adicionalmente, la misma presencia de sotobosque puede afectar la lluvia y depredación de semillas, ya sea aportando a la lluvia de semillas directamente o atrayendo dispersores y depredadores (Simonetti et al. 2012). Asimismo, el sotobosque genera condiciones de microhábitat diferentes a áreas sin sotobosque, principalmente aumentando la retención de humedad y disminuyendo la entrada de luz, lo cual puede modificar la probabilidad de germinación dependiendo de los requerimientos de las especies (Grubb 1977). Estos diferentes efectos del sotobosque sobre la regeneración de plantas nativas hacen muy difícil plantear predicciones respecto al efecto neto de este.

El centro-sur de Chile se caracteriza por la presencia de una gran superficie de plantaciones forestales, especialmente entre los 35 y $40^{\circ}$ de latitud Sur (Echeverría et al. 2006). Estas han llegado a cubrir el 3,1 \% del territorio nacional, de lo cual el 63,7 \% corresponde a la especie exótica $P$. radiata (INFOR 2011). Aunque la mayor parte de estas plantaciones se estableció en áreas donde el bosque nativo ya había sido eliminado o fuertemente degradado, una parte de estas se han establecido producto del reemplazo de bosque nativo. Así, actualmente, el paisaje de Chile centro-sur se presenta como un mosaico donde el bosque nativo se encuentra altamente fragmentado e inmerso en una matriz de plantaciones de $P$. radiata (Echeverría et al. 2006). Estas plantaciones presentan diferentes asociaciones vegetales y niveles de desarrollo de sotobosque bajo el dosel, desde sitios con abundante sotobosque hasta sitios sin más vegetación que los árboles plantados (Ramírez et al. 1984). Además, Becerra y Montenegro (2013) demuestran que diferentes especies nativas reclutan naturalmente bajo la cobertura de individuos naturalizados de $P$. radiata, e incluso la probabilidad de reclutamiento de especies nativas es muy similar entre parches de bosque nativo y sitios bajo cobertura de $P$. radiata, en ambos casos mayores que en sitios abiertos. Esto sugiere que $P$. radiata no inhibe la regeneración de especies nativas, $y$, por el contrario, la facilita en comparación a sitios abiertos.

En este trabajo se evalúa el efecto de la distancia al borde con bosque nativo sobre la lluvia de semillas y la depredación de semillas post-dispersión. Además se evalúa el efecto del sotobosque presente en las plantaciones, en la lluvia de semillas, depredación post-dispersión de semillas y germinación en una plantación forestal de $P$. radiata de la zona costera de Chile centro-sur. Respecto al efecto de la distancia al bosque nativo, se espera que la lluvia de semillas sea menor y la depredación de semillas fuera mayor a medida que aumenta la distancia al borde de la 
plantación con bosque nativo. Respecto del efecto del sotobosque, se espera que la lluvia de semillas y depredación de semillas fuera menor en plantaciones sin sotobosque, que en aquellas con sotobosque. Así mismo, se espera que la probabilidad de germinación sea menor en plantaciones sin sotobosque que en aquellas con sotobosque. Con esta información será posible establecer medidas de manejo tales como el ancho de los rodales de manera de asegurar la lluvia de semillas al interior de ellos, así como la importancia de aplicar restricciones que reduzcan la eliminación de sotobosque en los procesos de cosecha o manejo en las plantaciones de $P$. radiata.

\section{MÉTODOS}

El área de bosque Maulino Costero estudiada posee un clima templado, con precipitaciones estacionales que se concentran en la época invernal, variando anualmente entre 1.100-1.300 mm. El estudio se desarrolló a través de diversos experimentos llevados a cabo en plantaciones de $P$. radiata de la localidad de Tregualemu, región del Maule. Estas plantaciones fueron terrenos usados anteriormente para la agricultura y tienen al menos 20 años de edad (Vergara y Simonetti 2004). Los rodales de P. radiata seleccionados para este experimento (figura 1) son coetáneos y sometidos al mismo manejo de podas, raleos y tala rasa. Todos se presentaron en áreas relativamente planas
( $<5 \%$ pendiente) y presentaron una cobertura arbórea de aproximadamente un $80 \%$ en el período del estudio. Al momento del estudio todos los individuos de $P$. radiata estaban en edad reproductiva.

La distribución del sotobosque en estos rodales no es homogénea, sino que existen áreas con sotobosque con diferente grado de desarrollo y otras sin sotobosque, clasificadas como tal al no haber vegetación arbustiva a más de $50 \mathrm{~cm}$ del suelo. Las especies dominantes en zonas con mayor desarrollo de sotobosque son Aristotelia chilensis (Mol.) Stuntz, Peumus boldus Mol., Quillaja saponaria Mol. y Rubus ulmifolius Schott (Poch y Simonetti 2013). Aledaño a las plantaciones, existen fragmentos remanentes de bosque nativo dominados por Nothofagus glauca (Phil.) Krasser, Cryptocarya alba (Mol.) Looser, Aristotelia chilensis, Aextoxicon punctatum R. et P., Gevuina avellana Mol. (Grez et al. 2006). Las áreas con y sin sotobosque seleccionadas dentro de las plantaciones se situaron a diferentes distancias de los remanentes de bosque nativo (figura 1).

La densidad de $P$. radiata adultos plantados para sitios sin sotobosque no difirió significativamente con la densidad en sitios con sotobosque (U Mann-Whitney $=106,5$, $P=0,34$ ) (cuadro 1). La luminosidad a nivel del suelo fue mayor en sitios sin sotobosque, aunque las diferencias entre sitios con y sin sotobosque fueron solo marginalmente significativas (U Mann-Whitney $=13, P=0,055)$ (cuadro 1$)$.

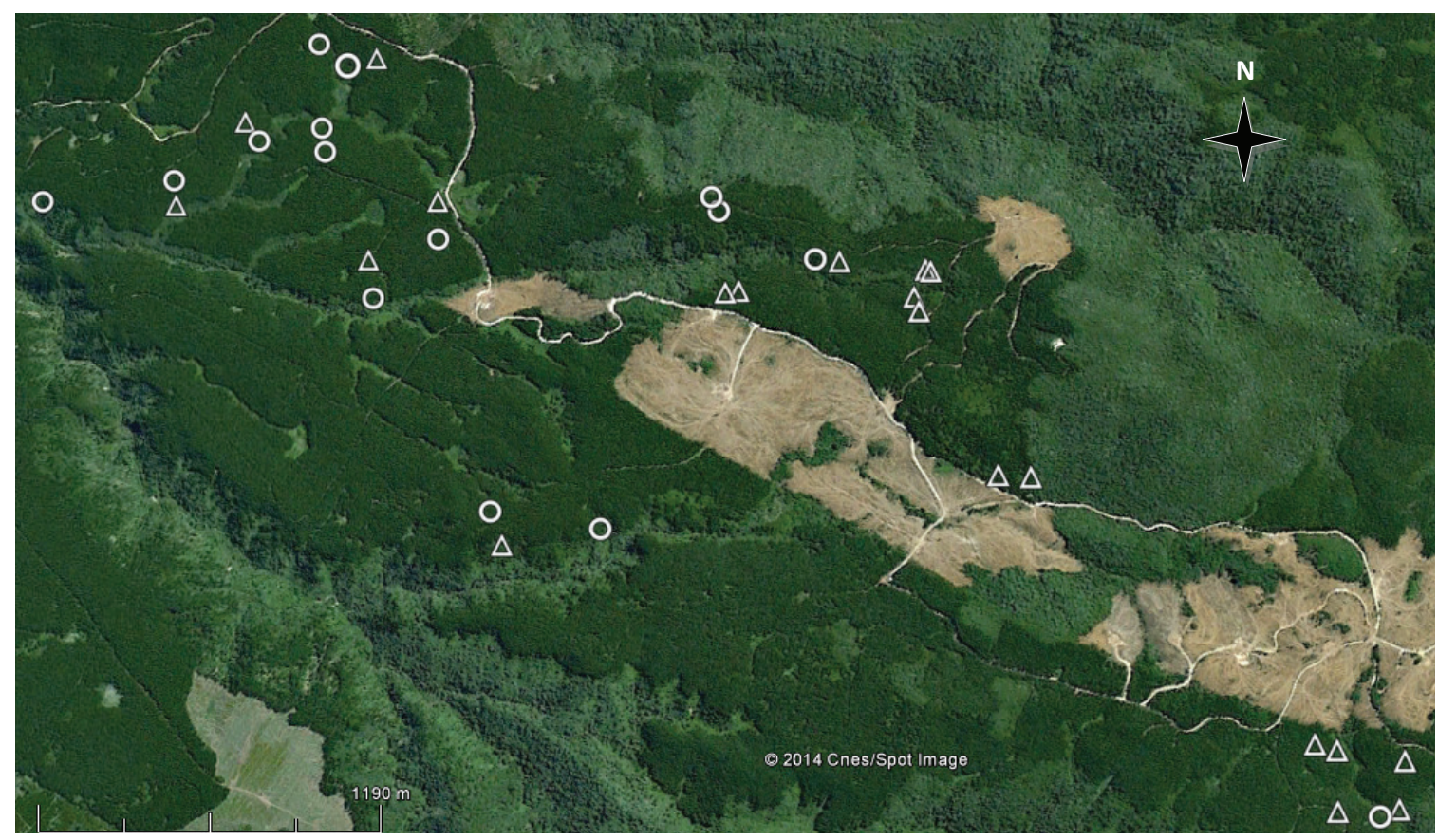

Figura 1. Imagen satelital del área de estudio. Las zonas boscosas más oscuras corresponden a plantaciones de $P$. radiata mientras que las zonas boscosas más claras a fragmentos de bosque nativo. Triángulos corresponden a los sitios de muestreo con sotobosque y círculos a sitios sin sotobosque.

Satellital image of the study area. Forest areas in dark correspond to Pinus radiata plantations, while forest areas in grey to native forest fragments. Triangles indicate sampling sites with understory and circles sites without understory. 
Lluvia de semillas. Para evaluar la lluvia de semillas se cuantificó la riqueza de especies y abundancia total de semillas de plantas leñosas capturadas en trampas de semillas, en 20 sitios dentro de un rodal de $P$. radiata con sotobosque y 20 sitios dentro de un rodal de $P$. radiata sin sotobosque. Estos sitios se eligieron al azar, cubriendo distancias al borde con bosque nativo desde 0 a $134 \mathrm{~m}$ en sitios con sotobosque y de 5 a $141 \mathrm{~m}$ en sitios sin sotobosque. Con el fin de evaluar la dependencia espacial entre los puntos de monitoreo de la lluvia de semillas, se realizó un prueba de Mantel construyendo una matriz de distancia euclidiana entre los distintos puntos muestreados, con los datos obtenidos en la lluvia de semillas y luego realizando una correlación con la distancia entre los puntos. Al no existir correlación entre los datos $(\mathrm{r}=0,005 ; P=0,96)$ se corroboró la independencia espacial de estos (Quinn y Keough 2002). Se ubicó una trampa de semillas en cada sitio. Cada trampa consistió de una canastilla de malla de 50 x $50 \mathrm{~cm}$, ubicada aproximadamente a 1 metro del suelo. Las semillas se recolectaron desde octubre de 2011 hasta octubre de 2012 (un año). Para los análisis, se eliminaron cuatro trampas de semillas, todas ellas ubicadas en sitios sin sotobosque, debido a que fueron derribadas por ganado presente en la zona.

Depredación de semillas y germinación. Para evaluar la depredación de semillas y germinación, se utilizó la especie esclerófila Quillaja saponaria. Esta especie se seleccionó debido a que facilitaba la evaluación de estos procesos y está presente tanto en plantaciones como en fragmentos de bosque nativo, aunque con una baja frecuencia. Por ello, los resultados de germinación se basan en una especie que puede regenerar en plantaciones. Además, las semillas de esta especie pueden ser depredadas por una amplia variedad de micromamíferos y no son dispersadas por aves. Esto permite que en el experimento de depredación este proceso no sea confundido con dispersión. Estas semillas se obtuvieron del Centro de Semillas, Facultad de Ciencias Forestales, Universidad de Chile.

Para los experimentos de depredación y germinación se establecieron 50 sitios al azar a diferentes distancias del bosque nativo, de los cuales 25 se ubicaron en un rodal con sotobosque y otros 25 en un rodal sin sotobosque, procurando una distancia entre puntos de al menos $10 \mathrm{~m}$ de separación. Para la depredación de semillas post-dispersión se instaló durante el mes de septiembre de 2012, una placa petri con 10 semillas de Q. saponaria, en cada uno de los 50 puntos seleccionados. Las semillas se adhirieron con pegamento a la placa Petri para evitar cualquier tipo de desplazamiento, por ejemplo por viento o agua, que no fuera causado intencionalmente por algún depredador. El registro de semillas depredadas se realizó en octubre de 2012, durante el cual se consideraron como depredadas aquellas semillas que presentaron marcas o que no se encontraron presentes sobre las placas, similar al protocolo desarrollado por Guerrero y Bustamante (2007). La proporción de semillas depredadas por placa se consideró como la probabilidad de depredación.

Para evaluar la probabilidad de germinación se instalaron 10 semillas de $Q$. saponaria durante el mes de julio de 2012, en cada uno de los 50 puntos seleccionados, obteniéndose un total de 250 semillas en sitios con sotobosque y 250 en sitios sin sotobosque. Sobre las 10 semillas se instaló una malla para evitar la depredación de las semillas y así cuantificar el efecto independiente de la germinación. El registro de semillas germinadas se llevó a cabo durante todos los meses hasta octubre de 2012. Se consideraron germinadas a las semillas que exhibían una radícula que había alcanzado una longitud de al menos $5 \mathrm{~mm}$ (McAlpine y Drake 2002).

Tanto para la depredación post-dispersión como para la germinación, la independencia espacial de los datos se corroboró mediante la prueba de Mantel $(\mathrm{r}=-0,02, P=$ 0,$60 ; \mathrm{r}=-0,02, P=0,52$, respectivamente), realizado de la misma manera que para la lluvia de semillas pero con los datos respectivos a depredación post-dispersión y germinación (Quinn y Keough 2002).

\section{RESULTADOS}

Lluvia de semillas. Del total de semillas recuperadas en las trampas con y sin sotobosque, se registraron 21 especies diferentes. De estas, 12 especies fueron leñosas, llegando a reconocer hasta el nivel de especie en 11 de ellas: Pinus radiata, Aristotelia chilensis, Muehlenbeckia hastulata (J.E. Sm.) Johnst., Genista monspessulana (L.) L.A.S. Johnson, Nothofagus obliqua (Mirb.) Oerst., Peumus boldus, Lithrea caustica (Mol.) H. et A., Proustia pyrifolia DC,

Cuadro 1. Medidas descriptivas de densidad de P. radiata plantados y de la luminosidad medida a nivel del suelo.

Descriptive values of Pinus radiata density and luminosity and ground level.

\begin{tabular}{ccccc}
\hline Variable & Sotobosque & Mediana & Mínimo & Máximo \\
\hline \multirow{2}{*}{ Densidad (individuos ha $^{-1}$ ) } & Con & 486 & 465 & 503 \\
& Sin & 470 & 453 & 489 \\
\hline \multirow{2}{*}{ Luminosidad (lux) } & Con & 6.470 & 2.330 & 9.920 \\
& Sin & 1.530 & 930 & 9.050 \\
\hline
\end{tabular}


Laurelia sempervirens (R. et P.) Tul., Quillaja saponaria y Senna stipulacea (Aiton) Irw. et Barneby. Además, se registró una especie de la familia Myrtaceae, que no se pudo identificar hasta el nivel de especie.

En total se registraron 1.100 semillas de especies leñosas, de las cuales 638 semillas correspondieron a P. radia$\mathrm{ta}$, mientras que 462 semillas correspondieron a distintas especies leñosas que se encuentran en el sotobosque de las plantaciones, en su mayoría nativas (con la excepción de G. monspessulana). Pinus radiata fue la especie más abundante en ambos tipos de sitio, con y sin sotobosque, luego A. chilensis, con 154 y 86 semillas, respectivamente. Esto corresponde al 47,1 \% y 58,5\%, respectivamente, del total de semillas de especies leñosas en cada tipo de sitio (total calculado sin considerar la especie $P$. radiata). En segundo lugar se encuentra $N$. obliqua en sitios con sotobosque (67 semillas en total) y G. monspessulana en sitios sin sotobosque ( 29 semillas en total). Esta última es la única especie que se encontró ausente en sitios con sotobosque, mientras que $L$. caustica, S. stipulacea, P. boldus y $Q$. saponaria se encontraron presentes solo en la lluvia de semillas de sitios con sotobosque.

Las semillas se distribuyeron a lo largo de todo el rango de distancia muestreado, encontrándose presente $P$. radiata a lo largo de todo el rango de distancia y en general siendo la más abundante de todas las especies presentes. Además se observó que la especie nativa $A$. chilensis, se encuentra presente en todo el rango de distancia muestreado (figura 2). Si bien pocas especies mostraron una clara tendencia entre la lluvia de semillas y distancia al borde con bosque nativo (figura 2), algunas especies efectivamente presentaron una reducción del número de semillas con la distancia al borde, por ejemplo, A. chilensis, Myrtaceae (figura 2A), L. caustica y P. pyrifolia (figura 2B).

Independiente de la distancia, la riqueza de especies fue mayor en sitios con sotobosque que en sitios sin sotobosque (cuadro 3), aunque las diferencias fueron marginalmente significativas (U Mann-Whitney $=99, P=0,053$ ). Sin embargo, la abundancia total de semillas no difirió entre sitios con y sin sotobosque (cuadro 3) (U Mann-Whitney $=122, P=0,236$ ).

A pesar de que pocas especies mostraron una relación negativa entre lluvia de semillas y distancia al borde del bosque nativo (figura 2), al considerar el conjunto de especies se observaron tendencias más claras. La relación entre la distancia al borde con bosque nativo y abundancia de semillas en sitios con sotobosque se ajustó significativamente a una función exponencial de la forma $\mathrm{y}=\exp$ $(-0,01896 \mathrm{x}+3,4306)(\mathrm{G}=8,44 ; P=0,004)$, existiendo una disminución muy rápida en la abundancia total de

Cuadro 3. Medidas descriptivas de la riqueza de especies y abundancia de semillas.

Descriptive values of species richness and abundance of seeds.

\begin{tabular}{lccccc}
\hline \multirow{2}{*}{ Variable } & Sotobosque & Mediana & Mínimo & Máximo & Total \\
\hline \multirow{2}{*}{ Riqueza } & Con & 2 & 0 & 6 & 10 \\
& Sin & 1 & 0 & 1 & 7 \\
\hline \multirow{2}{*}{ Abundancia } & Con & 8 & 0 & 77 & 327 \\
& Sin & 3 & 0 & 49 & 135 \\
\hline
\end{tabular}

Cuadro 2. Medidas descriptivas de la abundancia de semillas para cada especie leñosa presente en la lluvia de semillas.

Descriptive values of seed abundance for woody species recorded in the seed rain.

\begin{tabular}{lrrrrrrrr}
\hline \multirow{2}{*}{ Especie } & \multicolumn{9}{c}{ Con sotobosque } & \multicolumn{5}{c}{ Sin sotobosque } \\
\cline { 2 - 8 } & Mediana & Mínimo & Máximo & Total & Mediana & Mínimo & Máximo & Total \\
\hline Pinus radiata & 18,5 & 6 & 42 & 369 & 13 & 2 & 37 & 257 \\
Aristotelia chilensis & 4,5 & 0 & 38 & 154 & 3 & 0 & 19 & 79 \\
Nothofagus obliqua & 0 & 0 & 50 & 67 & 0 & 0 & 3 & 4 \\
Myrtaceae & 0 & 0 & 35 & 43 & 0 & 0 & 2 & 3 \\
Muehlenbeckia hastulata & 0,5 & 0 & 7 & 30 & 0 & 0 & 6 & 13 \\
Genista monspessulana & - & - & - & - & 0 & 0 & 29 & 29 \\
Proustia pyrifolia & 0 & 0 & 3 & 9 & 0 & 0 & 2 & 5 \\
Lithrea caustica & 0 & 0 & 6 & 13 & - & - & - & - \\
Senna stipulacea & 0 & 0 & 6 & 6 & - & - & - & - \\
Laurelia sempervirens & 0 & 0 & 2 & 2 & 0 & 0 & 1 & 2 \\
Peumus boldus & 0 & 0 & 1 & 2 & - & - & - & - \\
Quillaja saponaria & 0 & 0 & 1 & 1 & - & - & - & - \\
\hline
\end{tabular}



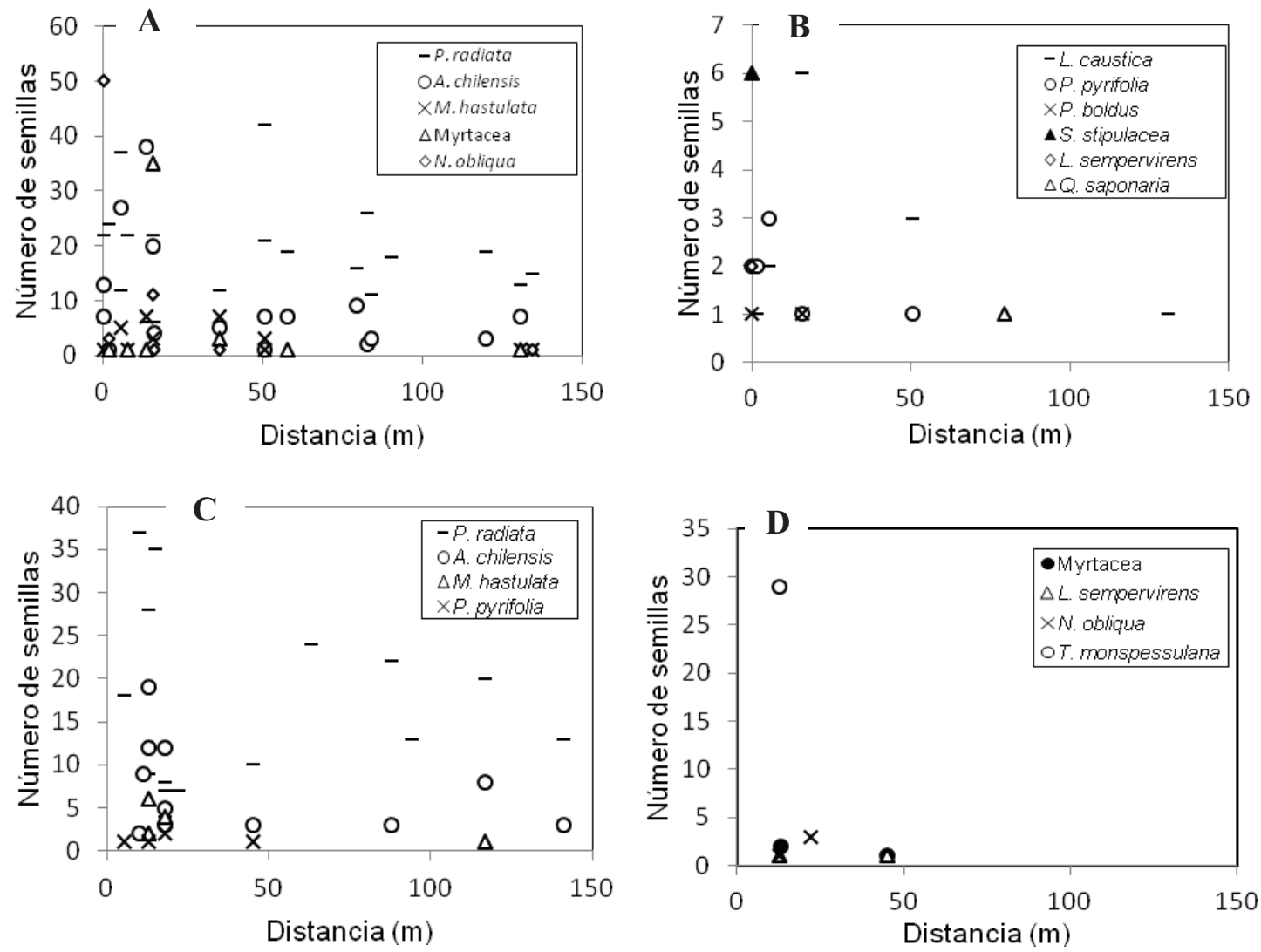

Figura 2. Número de semillas en función de la distancia al borde de la plantación en: sitios con sotobosque (A) especies que cayeron en las trampas en una proporción mayor a 0,25 respecto del total de semillas en la trampa y (B) especies que cayeron en las trampas en una proporción menor o igual a 0,25 ; sitios sin sotobosque $(C)$ especies que cayeron en las trampas en una proporción mayor o igual a 0,25 y (D) especies que cayeron en las trampas en una proporción menor a 0,25.

Number of seeds at different distances from the plantation edge at: sites with understory (a) species that fell on seed traps in a proportion over 0.25 respect to the total number of seeds in the trap, and (b) species that fell on seed traps in a proportion lower than or equal to 0.25 ; sites without understory (c) species that fell on seed traps in a proportion higher than 0.25 and (d) species that fell on seed traps in a proportion inferior or equal to 0.25 .

semillas en los primeros 40 metros, luego de los cuales disminuyó más suavemente (figura 3A). Similarmente, la riqueza de especies en sitios con sotobosque (figura 3B) se correlacionó negativa y linealmente con la distancia al borde de bosque nativo $\left(\mathrm{r}_{\mathrm{s}}=-0,44, P=0,05\right)$.

En cambio, en sitios sin sotobosque no se detectó ningún tipo de relación significativa entre la distancia y la abundancia (figura 4A) o riqueza de especies (figura 4B) ( $\mathrm{r}_{\mathrm{s}}=-0,16, P=0,56 ; \mathrm{r}_{\mathrm{s}}=-0,19, P=0,48$, respectivamente). Incluso en el rango donde se concentraron nueve de las 17 trampas (de 0 a $20 \mathrm{~m}$ ), solo en tres de ellas cayeron más de 10 semillas y la probabilidad de encontrar más de 10 semillas en una trampa fue de 0,19 (figura 4).

Depredación post-dispersión y germinación. La probabilidad de depredación post-dispersión presentó una mediana de 0,2 en sitios con sotobosque y de 0,7 en sitios sin sotobosque (rango de 0 a 1 en ambos sitios), no existiendo diferencias significativas entre ellos (U Mann-Whitney = $236, P=0,14)$. Además, la depredación post-dispersión no se correlacionó con la distancia al borde con bosque nativo en sitios con sotobosque $\left(\mathrm{r}_{\mathrm{s}}=0,03, P=0,87\right)$, ni en sitios sin sotobosque $\left(\mathrm{r}_{\mathrm{s}}=-0,02, P=0,94\right)$.

Utilizando la muestra total para calcular la probabilidad de germinación de semillas de $Q$. saponaria en sitios con sotobosque, esta fue de 0,66 $(n=208)$, mientras que en sitios sin sotobosque fue de $0,39(n=116)$. La probabilidad de germinación fue significativamente mayor en plantaciones con sotobosque que sin sotobosque (Prueba de Fisher $\mathrm{Z}=5,62, P<0,001)$.

\section{DISCUSIÓN}

Los resultados obtenidos en este estudio sugieren que la riqueza y abundancia de la lluvia de semillas al interior de las plantaciones está limitada por la distancia al borde con el bosque nativo, aunque solo en sitios de plantaciones con sotobosque. En sitios sin sotobosque, en cambio, no se observó correlación entre la lluvia de semillas y la distancia, lo cual sugiere que el sotobosque influye en la 

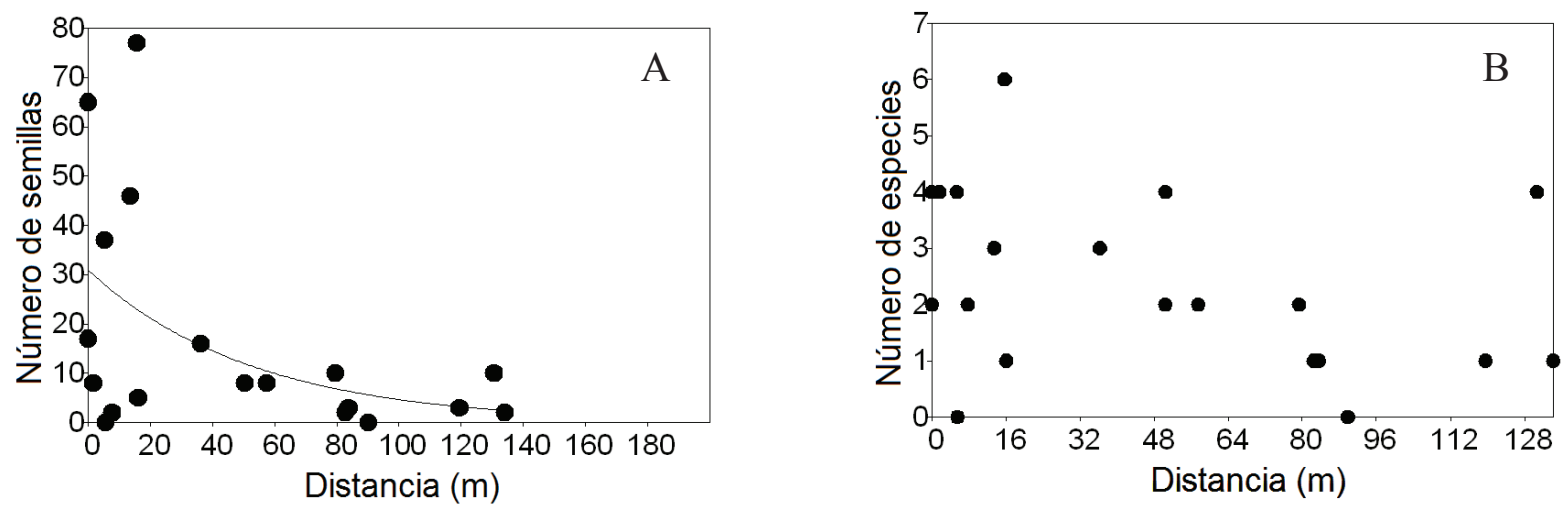

Figura 3. Relación de la abundancia y riqueza de especies en lluvia de semillas con la distancia al bosque nativo, en plantaciones con sotobosque. (A) Abundancia, expresado como número de semillas, línea de regresión ajustada mediante la función y = exp $(-0,01896 x+3,4306)$. (B) Riqueza, expresado como número de especies.

Relationship of species richness and abundance in seed rain with distance to native forest, in plantations with understory: (a) abundance (number of seeds); (b) species richness (number of woody species).
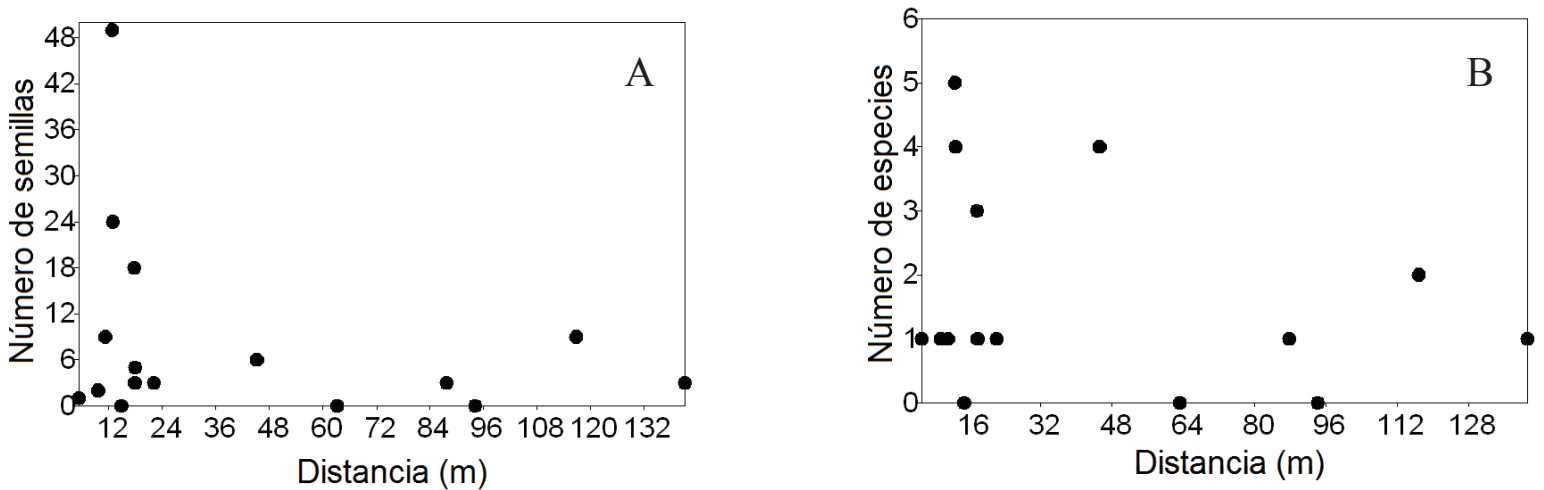

Figura 4. Relación de la abundancia y riqueza de especies en la lluvia de semillas con la distancia al bosque nativo, en plantaciones sin sotobosque. (A) abundancia, expresado como el número de semillas. (B) riqueza, expresado como número de especies.

Relationship of species richness and abundance in seed rain with distance to native forest, in plantations without understory: (a) abundance (number of seeds); (b) species richness (number of woody species).

dispersión de semillas. Es posible que en sitios con sotobosque exista mayor presencia de dispersores de semillas en los primeros metros desde el borde con bosque nativo, y que luego estos disminuyan hacia el interior de las plantaciones como ocurre con algunas aves (Simonetti et al. 2012). Esto podría generar una mayor lluvia de semillas en los bordes que en el interior. Por ejemplo, esto podría estar ocurriendo en semillas de $L$. caustica y algunas mirtáceas, cuya abundancia se correlaciona con la distancia, pero casi no se encuentran presentes en sitios sin sotobosque. En sitios sin sotobosque, A. chilensis es de las pocas especies presentes y la más abundante, pero su abundancia no presenta relación con la distancia al borde con el bosque nativo, distribuyéndose a lo largo de todo el rango de distancia. Así, la ausencia de especies cuya lluvia de semillas disminuya con la distancia, más la presencia de $A$. chilensis en todas las distancias desde el borde, determinan que en sitios sin sotobosque no exista una relación entre abundancia de semillas y distancia al bosque nativo.

Por otro lado, si bien existe una tendencia de la abundancia de semillas a ser mayor en sitios con que sin sotobosque, las diferencias no fueron estadísticamente significativas. Es decir, la presencia de sotobosque y las semillas que puede producir, no incrementan significativamente la lluvia de semillas al interior de las plantaciones. Esto sugiere que el principal aporte de semillas proviene desde el bosque nativo colindante. Sin embargo, la lluvia de semillas sí tiene significativamente menos especies en sitios sin sotobosque, lo cual puede deberse a un aporte directo de semillas de las especies presentes en el sotobosque, o a la atracción de dispersores de una mayor variedad de especies desde los fragmentos de bosque nativo (Simonetti et 
al. 2012). Así, el efecto del sotobosque no sería suficiente para aumentar la abundancia de semillas, pero sí aportaría con especies que, de no estar presente el sotobosque, no llegarían al interior de las plantaciones.

La depredación de semillas post-dispersión no varía con la distancia al bosque nativo. Esto sugiere que la abundancia de depredadores de semillas es similar a diferentes distancias del bosque nativo, lo que es consistente con la similar abundancia de micromamíferos consumidores de semillas entre plantaciones de $P$. radiata y fragmentos de bosque nativo observado en este mismo paisaje forestal (Saavedra y Simonetti 2005). Además, la depredación no difiere entre sitios con y sin sotobosque, lo que sugiere que el sotobosque no influye en los tipos de depredadores de semillas, o que si las especies de depredadores difieren entre sitios con y sin sotobosque, la abundancia podría ser similar.

La germinación fue positivamente afectada por la presencia de sotobosque. Esto podría deberse a condiciones microambientales particulares que se forman en sitios con sotobosque. Por ejemplo, existe una menor luminosidad en sitios con sotobosque a pesar que la densidad de $P$. radiata no varió entre sitios con y sin sotobosque. Esto puede ser de gran relevancia para la regeneración, ya que generalmente menor luminosidad se asocia con mayor humedad del suelo (Wiberg 1991). Aunque $Q$. saponaria ha sido catalogada como sombra-intolerante en estado juvenil o adulto, una baja luminosidad no sería limitante para su germinación. Muchas especies leñosas en Chile central requieren de un dosel que genere especialmente mayor humedad del suelo, entre otras condiciones, para poder reclutar (Holmgren et al. 2000, Becerra y Montenegro 2013). De esta manera, el dosel del sotobosque podría estar incrementando la humedad del suelo y con ello la germinación, incluso en una especie sombra-intolerante como Q. saponaria. Además, en sitios sin sotobosque del área el $\mathrm{pH}$ del suelo es significativamente más ácido que sitios con sotobosque producido por las acículas que caen desde el dosel, lo cual también podría limitar la germinación ${ }^{1}$. Sin embargo, las condiciones de suelo generadas por $P$. radiata no parecen afectar fuertemente la regeneración de especies leñosas, o si lo hace, es similar al efecto generado por parches de bosque nativo (Becerra y Montenegro 2013). Así, la existencia de individuos juveniles y adultos de esta especie habitando el interior de plantaciones (Becerra y Simonetti, datos no publicados), sugiere que puede llegar a establecerse al interior de las plantaciones. Por lo tanto, es más probable aún que especies más tolerantes a la sombra puedan regenerarse y formar parte de un sotobosque desarrollado en el interior de plantaciones (Becerra y Montenegro 2013).

En un paisaje fragmentado como el de la zona costera de Chile centro-sur, donde existen pocas reservas de

Simonetti JA, CF Estades, M Pérez, AA Grez. Does the understory compete with adult Monterey pines (Pinus radiata): an experimental test. Ciencia e Investigación Agraria (en revisión). bosque nativo (Echeverría et al. 2006), los fragmentos de bosque nativo que aún quedan, son capaces de conservar procesos y especies (Grez et al. 2006). Los resultados sugieren que el beneficio para la biodiversidad de remanentes de bosque nativo puede extenderse más allá de sus límites, por ejemplo en las plantaciones de $P$. radiata presentes en la matriz que los rodea (e.g. Brudvig et al. 2009, Craig et al. 2011). Los resultados observados en este trabajo sugieren que la lluvia de semillas en plantaciones depende en gran medida de la distancia a los fragmentos que producen semillas. Además, la presencia de sotobosque probablemente mejora la riqueza de la lluvia de semillas y las condiciones de reclutamiento. Por lo tanto, el manejo de las plantaciones, especialmente en las etapas de raleos y talas, debiera considerar acciones que permitan mantener el sotobosque de manera de continuar incrementando la diversidad de este al establecerse las plantaciones. Basándose en la respuesta de la abundancia de la lluvia de semillas en sitios con sotobosque, es posible realizar una proyección de la curva de dispersión. Esta proyección indica que alrededor de los $160 \mathrm{~m}$ llegarán muy pocas o ninguna semilla, valor similar o mayor a los obtenidos en otros estudios (Hewitt y Kellman 2002). De esta manera, los rodales de plantación debieran tener un ancho máximo de no más de $320 \mathrm{~m}$ entre fragmentos de bosque nativo, de manera de asegurar continuidad una lluvia de semillas a lo ancho de todo el rodal de plantación y con ello recuperar el sotobosque después de la tala rasa que normalmente se realiza en las plantaciones.

En conclusión, la lluvia de semillas hacia el interior de las plantaciones es afectada por la distancia al bosque nativo, aunque esto ocurre solo con la presencia de sotobosque, sugiriendo que las semillas provienen principalmente de los fragmentos de bosque nativo aledaño. Además, el sotobosque incrementa la lluvia de semillas, no afecta la depredación de semillas e incrementa la probabilidad de germinar. En consecuencia, el sotobosque aumenta la probabilidad de regeneración de especies nativas dentro de plantaciones. Así, la preservación de los remanentes de bosque nativo en paisajes dominados por plantaciones forestales debe ser prioridad para que el aporte de las plantaciones a la biodiversidad sea efectivo, ya que de estos provienen gran parte de las semillas que generan el sotobosque y biodiversidad al interior de las plantaciones. Sin comprometer la producción forestal, relevante un país como Chile donde el 3,1 \% del PIB nacional proviene de esta actividad (INFOR 2011), es posible tomar medidas de manejo dirigidas a conservar y facilitar la presencia de sotobosque (Brockerhoff et al. 2008), de manera de lograr una conservación de la biodiversidad a escala de paisaje, y así cumplir con los requerimientos internacionales y compromisos adquiridos por el país (FSC 2002, CBD 1992).

\section{AGRADECIMIENTOS}

Este trabajo ha sido realizado con el apoyo de Fondecyt 1095046. Agradecemos a la Corporación Nacional Fores- 
tal y Forestal Masisa S.A. por permitirnos trabajar en sus predios. El apoyo de R. Zuñiga en los trabajos de campo es muy agradecido. PB agradece al proyecto FB 0002-2014.

\section{REFERENCIAS}

Becerra P, G Montenegro. 2013. The widely invasive tree Pinus radiata facilitates regeneration of native woody species in a semi-arid ecosystem. Applied Vegetation Science 16:173-183.

Brockerhoff EG, CE Ecroyd, AC Leckie, MO Kimberley. 2003. Diversity and succession of adventive and indigenous vascular understory plants in Pinus radiata plantation forests in New Zealand. Forest Ecology and Management 185: 307-326.

Brockerhoff EG, H Jactel, JA Parrotta, CP Quine, J Sayer. 2008. Plantation forests and biodiversity: oxymoron or opportunity? Biodiversity and Conservation 17(5):925-951.

Brudvig LA, EL Damschen, JJ Tewksbury, NM Haddad, DJ Levey. 2009. Landscape connectivity promotes plant biodiversity spillover into non-target habitats. Proceedings of the National Academy of Sciences of the USA 106(23): 9328-9332.

CBD (Convention on Biological Diversity, CA). 1992. Convention on biological diversity. CBD, Montreal. Consultado 15 oct. 2013. Disponible en https://www.cbd.int/convention/

Craig MT, JL Orrock, LA Brudvig. 2011. Edge-mediated patterns of seed removal in experimentally connected and fragmented landscapes. Landscape Ecology 26(10): 1373-1381.

Echeverría C, D Coomes, J Salas, J Rey-Benayas, A Lara, A Newton. 2006. Rapid deforestation and fragmentation of Chilean temperate forests. Biological Conservation 130(4): 481-494.

FSC (Forestal Stewardship Council). 2002. FSC principles and criteria for forest stewardship. Consultado 21 de noviembre 2013. Disponible en http://www.cl.fsc.org/fileadmin/ fsc_chile/page_content/pdf/FSC_STD_01_001_V4_0 EN_FSC_Principles_and_Criteria.pdf

Gonzales RS, T Nagashizuka. 2010. Broad-leaf species composition in Cryptomeria japonica plantations with respect to distance from natural forest. Forest Ecology and Management 259(10): 2133-2140.

Grez AA, JA Simonetti, RO Bustamante. 2006. Biodiversidad en ambientes fragmentado de Chile: patrones y procesos a diferentes escalas. Santiago, Chile. Editorial Universitaria. $232 \mathrm{p}$.

Grubb PJ. 1977. The maintenance of species-richness in plant communities: the importance of the regeneration niche. Biological Reviews 52(1): 107-145.

Guerrero PC, RO Bustamante. 2007. Can native tree species regenerate in Pinus radiata plantations in Chile? Evidence from field and laboratory experiments. Forest Ecology and Management 253(1): 97-102.

Hewitt N, M Kellman. 2002. Tree seed dispersal among forest fragments: II. Dispersal abilities and biogeographical controls. Journal of Biogeography 29(3): 351-363.

Holmgren M, AM Segura, ER Fuentes. 2000. Limiting mechanisms in the regeneration of the Chilean matorral. Plant Ecology 147(1): 49-57.

INFOR (Instituto Forestal, CL). 2011. El sector forestal chileno 2011. Consultado 10 oct. 2013. Disponible en http://web. ffm.cl/index.php?option $=$ com jdownloads $\&$ Itemid $=5 \&$ vie $\mathrm{w}=$ finish \& cid $=152 \&$ catid $=8$

McAlpine KG, DR Drake. 2002. The effects of small-scale environmental heterogeneity on seed germination in experimental treefall gaps in New Zealand. Plant Ecology 165(2): 207-215.

Poch TJ, JA Simonetti. 2013. Insectivory in Pinus radiata plantations with different degree of structural complexity. Forest Ecology and Management 304: 132-136.

Quinn GP, MJ Keough. 2002. Experimental design and data analysis for biologists. Cambridge, England. Cambridge University Press. 537 p.

Ramírez C, H Figueroa, R Carrillo, D Contreras. 1984. Estudio fitosociológico de los estratos inferiores de un bosque de pino (Valdivia, Chile). Bosque 5(2): 65-81.

Saavedra B, JA Simonetti. 2005. Small mammals of Maulino forest remnants, a vanishing ecosystem of south-central Chile. Mammalia 69(3-4): 337-348.

Simonetti JA, AA Grez, CF Estades (eds.). 2012. Biodiversity conservation in agroforestry landscapes: challenges and opportunities. Santiago, Chile. Editorial Universitaria. 154 p.

Utsugi E, H Kanno, N Ueno, M Tomita, T Saitoh, M Kimura, K Kanou, K Seiwa. 2006. Hardwood recruitment into conifer plantations in Japan: effects of thinning and distance from neighboring hardwood forests. Forest Ecology and Management 237(1): 15-28.

Vergara PM, JA Simonetti. 2004. Avian responses to fragmentation of the Maulino Forest in Central Chile. Oryx 38(4): 383-388.

Wiberg SC. 1991. Factores que influyen en la germinación y producción de plantas de quillay (Quillaja saponaria Mol.). Tesis de Licenciatura Ingeniero Forestal. Santiago, Chile. Universidad de Chile. 133 p. 
